\title{
Electroweak phase transition, gravitational waves and dark matter in two scalar singlet extension of the standard model
}

\author{
Vahid Reza Shajiee $^{\mathrm{a}}{ }^{\mathbb{D}}$, Ali Tofighi ${ }^{\mathrm{b}}$ \\ Department of Physics, Faculty of Basic Sciences, University of Mazandaran, Babolsar, Iran
}

Received: 28 February 2019 / Accepted: 13 April 2019 / Published online: 25 April 2019

(C) The Author(s) 2019

\begin{abstract}
In this paper, the electroweak phase transition, the gravitational waves and the dark matter issues are investigated in two scalar singlet extension of the standard model. The detectability of the gravitational wave signals are discussed by comparing the results with the sensitivity curves of eLISA, ALIA, DECIGO and BBO detectors. It is shown that the results support the recent reports on the dark matter relic density by Planck 2018 collaboration and the direct detection experiment by XENON1T2018 collaboration.
\end{abstract}

\section{Introduction}

The failure of the Standard Model (SM), in describing phenomena like the baryon asymmetry of the universe (BAU) and the dark matter (DM), brings to mind that the SM cannot be considered as a fundamental model. Nevertheless, the discovery of the Higgs boson [1,2] as the first observed scalar has opened the way to consider the SM as an effective field theory (EFT) and also a window to the Higgs portal. To address the BAU and the DM problems, many theories and models have been proposed beyond the SM such as supersymmetry studies [3-17]. Due to the attraction of the Higgs portal, it has been always of interest to investigate the SM extensions which directly challenge the Higgs portal like multi-scalar extensions [18-30]. The existence of interactions between the Higgs and new scalars makes such models reasonable for explaining the BAU, which needs a strong first-order electroweak phase transition (SFOEWPT), the gravitational waves $(\mathrm{GW})$ produced by an SFOEWPT and the DM. Moreover, such models also have other benefits. First, they are simple and straightforward. Second, they may be renormal-

\footnotetext{
a e-mail: v.shajiee@stu.umz.ac.ir

be-mail: a.tofighi@umz.ac.ir
}

ized, so no new physics scale is needed. Third, they may be gauge independent, if there exists a barrier in the potential at tree-level [31].

To justify the BAU, there is a need for Baryogenesis to exist [32-36] which itself needs an SFOEWPT, i.e. $\frac{v_{c}}{T_{c}} \gtrsim 1$ where $v_{c}$ is the Higgs vacuum expectation value $(\mathrm{VeV})$ at critical temperature $T_{c}$. This would not happen in the SM, but adding one or more new scalars to the SM potential may lead to an SFOEWPT. With regard to the new potential structure, two different phase transitions (PT) can happen. One of them is one-step PT in which there only exist initial and final phases. Cooling down the universe, it goes through a phase transition and breaks the electroweak symmetry. The other one is two-step (or multi-step) PT in which there also exists an intermediate phase (or more) between initial and final phases [37-41]. The reader is referred to [42-49] for the most recent studies on the EWPT.

During the SFOEWPT, the bubbles with the non-zero VeV nucleates in the plasma. The stochastic GW background arising from the SFOEWPT can be generated by the bubbles collisions and shocks [50-55], the sound waves [56-59], and the Magnetohydrodynamic (MHD) turbulence [60-64] in the plasma. Since the EWPT in the SM is a cross-over instead of strong one, the SM cannot predict the GW produced by the EWPT. So, this is another reason to look for beyond the SM. The recent observations of astrophysical GW [65-72] have brought the hope to detect the GW produced by the EWPT [73-75]. The reader is referred to [42,44-48,76-79] for the most recent studies on the GW produced by the EWPT.

As mentioned before, the SM cannot explain DM which existence is well established by cosmological evidence. As the simplest way, this incompetence can be justified by adding one (or more) gauge singlet scalar to the SM. Since the DM should be stable to provide the observed relic density $\Omega_{c} h^{2}=0.120 \pm 0.001$ by Planck 2018 [80], it is necessary to impose a discrete symmetry on the DM candidate, in present study $S_{2} \rightarrow-S_{2}$. On the other hand, the global minimum 
of potential at zero temperature spontaneously breaks this discrete symmetry, so necessarily $<S_{2}>=0$. The reader is referred to [43-45,47,49,81-86] for the most recent studies on the DM.

The present work is arranged as follows: in Sect. 2, the most general and renormalizable extension of the SM is presented by adding two scalar sectors $S_{1}$ and $S_{2}$ to the usual SM potential. ${ }^{1}$ Assigning a non-zero VeV to $S_{1}$, the SFOEWPTH can occur in the model. Imposing a $Z_{2}$ symmetry on $S_{2}$ makes it a viable candidate for the DM. Also, constraints on the parameter space are discussed. The EWPT, GW and DM are respectively investigated in Sects. 3, 4 and 5. Finally, some conclusions are presented in Sect. 6.

\section{The model}

The tree-level potential of the model is given by

$$
\begin{aligned}
V= & -m^{2} H^{\dagger} H+\lambda\left(H^{\dagger} H\right)^{2}+\kappa_{0} S_{1} \\
& +2\left(\kappa_{1} S_{1}+\kappa_{2} S_{1}^{2}+\kappa_{3} S_{2}^{2}\right) H^{\dagger} H \\
& +\frac{1}{2} m_{1}^{2} S_{1}^{2}+\frac{\lambda_{1}}{4} S_{1}^{4}+\kappa_{4} S_{1}^{3} \\
& +\frac{1}{2} m_{2}^{2} S_{2}^{2}+\frac{\lambda_{2}}{4} S_{2}^{4}+\kappa_{5} S_{1} S_{2}^{2},
\end{aligned}
$$

The potential 2.1 is the usual SM potential with two extra gauge singlet scalars and interaction terms which provide Higgs portal between the new scalars and the usual SM particles. $\mathrm{H}$ stands for the complex Higgs doublet, $H=$ $\left(\begin{array}{c}\chi_{1}+i \chi_{2} \\ \frac{1}{\sqrt{2}}\left(h+i \chi_{3}\right)\end{array}\right) \cdot S_{2}$ stands for the DM imposing $S_{2} \rightarrow-S_{2}$. Acquiring a non-zero $\mathrm{VeV}, S_{1}$ improves the strength of EWPT. The linear term of $S_{1}$ can be neglected by a shift in the potential. The $Z_{2}$ symmetry forbids the existence of linear and cubic terms for $S_{2}$, so Eq. (2.1) is the most general renormalizable potential which could be made by adding two new scalars. In the unitary gauge at zero temperature, the theoretical fields can be reparameterized in terms of the physical fields,

$$
H=\left(\begin{array}{c}
0 \\
\frac{1}{\sqrt{2}}(h+v)
\end{array}\right), S_{1}=s_{1}+\chi, \quad S_{2}=s_{2},
$$

where $v=246.22(\mathrm{GeV})$ and $\chi$ are the Higgs and $S_{1} \mathrm{VeV}$, respectively. Without loss of generality, one can write

\footnotetext{
1 The model first presented in [87] without the GW and the DM discussions. Here, the results of [87] are improved for the EWPT, also, the GW and the DM signals are investigated.
}

$$
\begin{aligned}
V= & -\frac{1}{2} m^{2} h^{2}+\frac{\lambda}{4} h^{4}+\left(\kappa_{1} s_{1}+\kappa_{2} s_{1}^{2}+\kappa_{3} s_{2}^{2}\right) h^{2} \\
& +\frac{1}{2} m_{1}^{2} s_{1}^{2}+\frac{\lambda_{1}}{4} s_{1}^{4}+\kappa_{4} s_{1}^{3} \\
& +\frac{1}{2} m_{2}^{2} s_{2}^{2}+\frac{\lambda_{2}}{4} s_{2}^{4}+\kappa_{5} s_{1} s_{2}^{2} .
\end{aligned}
$$

In order to have a stable potential, it is required that $[22,87]$

$\lambda>0, \quad \lambda_{1}>0, \quad \lambda_{2}>0, \quad \kappa_{2}>-\frac{\sqrt{\lambda \lambda_{1}}}{2}, \quad \kappa_{3}>-\frac{\sqrt{\lambda \lambda_{2}}}{2}$.

The tadpole equations at $(v, \chi, 0)$ read

$$
\begin{aligned}
& m^{2}=\lambda v^{2}+2\left(\kappa_{1} \chi+\kappa_{2} \chi^{2}\right), \\
& m_{1}^{2}=-\lambda_{1} \chi^{2}-3 \kappa_{4} \chi-2 \kappa_{2} v^{2}-\frac{\kappa_{1} v^{2}}{\chi} .
\end{aligned}
$$

From the diagonalization of squared-mass matrix and the tadpole equations, one can get

$$
\begin{aligned}
\lambda & =\frac{M_{1}^{2} \sin ^{2}(\theta)+M_{H}^{2} \cos ^{2}(\theta)}{2 v^{2}}, \\
\kappa_{2} & =\frac{\left(M_{H}^{2}-M_{1}^{2}\right) \sin (2 \theta)}{8 v \chi}-\frac{\kappa_{1}}{2 \chi}, \\
\lambda_{1} & =\frac{1}{2 \chi^{2}}\left(M_{1}^{2} \cos ^{2}(\theta)+M_{H}^{2} \sin ^{2}(\theta)+\frac{\kappa_{1} v^{2}}{\chi}-3 \chi \kappa_{4}\right), \\
m_{2}^{2} & =M_{2}^{2}-2 \kappa_{3} v^{2}-2 \kappa_{5} \chi,
\end{aligned}
$$

where $M_{H}=126(\mathrm{GeV}), M_{1}, M_{2}$ and $\theta$ are the Higgs mass, ${ }^{2}$ the physical mass of $S_{1}$, the physical mass of $S_{2}$ (the DM mass) and the mixing angle, respectively. In Ref. [89], by performing a global fit to the Higgs data from both ATLAS and CMS, the constraint on the mixing angle was given $|\theta| \leq 32.86^{\circ}$ at $95 \%$ confidence level (CL). In Ref. [90], by performing a universal Higgs fit, the upper limit on the mixing angle was given $|\theta| \leq 30.14^{\circ}$ at $95 \%$ CL. In the present work, a Monte Carlo scan is performed over the parameter space with

$$
\begin{aligned}
& 5 \mathrm{GeV} \leq M_{1} \leq 750 \mathrm{GeV}, \quad 5 \mathrm{GeV} \leq M_{2} \leq 750 \mathrm{GeV}, \\
& -23^{\circ} \leq \theta \leq 23^{\circ}, \\
& -80 \mathrm{GeV} \leq \kappa_{1} \leq 80 \mathrm{GeV}, \quad 0.0001 \leq \kappa_{3} \leq 0.1, \\
& -80 \mathrm{GeV} \leq \kappa_{4} \leq 80 \mathrm{GeV}, \\
& -80 \mathrm{GeV} \leq \kappa_{5} \leq 80 \mathrm{GeV}, \quad 30 \mathrm{GeV} \leq \chi \leq 120 \mathrm{GeV}, \\
& 0 \leq \lambda_{2} \leq 4 .
\end{aligned}
$$

\footnotetext{
2 The last announcement for the Higgs mass is $M_{H}=$ $125.09(\mathrm{GeV})$ [88], however, $1-3 \mathrm{GeV}$ deviation is acceptable.
} 


\section{Electroweak phase transition}

To investigate the EWPT in a model, one needs the finite temperature effective potential given by

$V_{\text {eff }}=V_{\text {tree-level }}+V_{1-\text { loop }}^{T=0}+V_{1-\text { loop }}^{T \neq 0}$,

where $V_{\text {tree-level }}, V_{1-\text { loop }}^{T=0}$ and $V_{1-\text { loop }}^{T \neq 0}$ are the tree-level potential (2.3), the one-loop corrected potential at zero temperature (the so-called Coleman-Weinberg potential) and the one-loop finite temperature corrections, respectively. The last two read

$$
\begin{aligned}
& V_{1-\text { loop }}^{T=0}= \pm \frac{1}{64 \pi^{2}} \sum_{i=h, s_{1}, s_{2}, W, Z, t} n_{i} m_{i}^{4}\left[\log \frac{m_{i}^{2}}{Q^{2}}-C_{i}\right], \\
& V_{1-\text { loop }}^{T \neq 0}=\frac{T^{4}}{2 \pi^{2}} \sum_{i=h, s_{1}, s_{2}, W, Z, t} n_{i} J_{ \pm}\left[\frac{m_{i}^{2}}{T^{2}}\right], \\
& J_{ \pm}\left(\frac{m_{i}^{2}}{T^{2}}\right)= \pm \int_{0}^{\infty} d y y^{2} \log \left(1 \mp e^{-\sqrt{y^{2}+\frac{m_{i}^{2}}{T^{2}}}}\right)
\end{aligned}
$$

where $n_{i}, m_{i}, Q$ and $C_{i}$ denote the degrees of freedom, the field-dependent masses, the renormalization scale and the numerical constants, respectively. The degrees of freedom and the numerical constants are respectively given by $\left(n_{h, s_{1}, s_{2}}, n_{W}, n_{Z}, n_{t}\right)=(1,6,3,12)$ and $\left(C_{W, Z}, C_{h, s_{1}, s_{2}, t}\right)$ $=(5 / 6,3 / 2)$. The upper (lower) sign is for bosons (fermions). Assuming the longitudinal gauge bosons polarizations are screened by plasma, thermal masses just contribute to the scalars, so Daisy corrections become small and can be neglected. There are three possibilities to deal with the renormalization scale $Q$. First one is to add some counter terms to the effective potential (3.1) to make it independent of Q without shifting $\mathrm{VeV}$ at zero temperature [91,92]. Second one is to set $\mathrm{Q}$ at a proper scale, like $Q=160(\mathrm{GeV})$ the running value of the top mass, $Q=246.22(\mathrm{GeV}) \mathrm{EW}$ scale and $Q=1(\mathrm{TeV})$ for supersymmetry purposes. Third one is to take $\mathrm{Q}$ as a free parameter to avoid shifting $\mathrm{VeV}$ at zero temperature. Here, the last one is considered.

The main idea of the EWPT is that the early universe, which from particle physics point of view may be described by potential (3.1), is in a high phase ${ }^{3}$ with $V e V=(<h>$ $,<s 1>,<s 2>)^{\text {high }}$ at high temperatures. Cooling down the universe, a new phase appears with $\mathrm{VeV}=(<h>$ $,<s 1>,<s 2>)^{l o w}$. As the universe cools down, the two phases become degenerate at the critical temperature $T_{c}$. Since the strength of the EWPT is governed by $\xi=\frac{v_{c}}{T_{c}}$,

\footnotetext{
${ }^{3}$ In this work, the high (low) phase denotes a phase which is the unstable (stable) vacuum for temperatures below $\mathrm{T}_{\mathrm{c}}$.
}

all that needs to be done is to calculate $v_{c}$ and $T_{c}$ from the following conditions:

$$
\begin{aligned}
& \left.\frac{\partial V_{e f f}}{\partial h}\right|_{(<h>,<s 1>,<s 2>)^{h i g h}, T=T_{c}}=0, \\
& \left.\frac{\partial V_{e f f}}{\partial h}\right|_{(<h>,<s 1>,<s 2>)^{l o w}, T=T_{c}}=0, \\
& \left.\frac{\partial V_{e f f}}{\partial s_{1}}\right|_{(<h>,<s 1>,<s 2>)^{h i g h}, T=T_{c}}=0, \\
& \left.\frac{\partial V_{e f f}}{\partial s_{1}}\right|_{(<h>,<s 1>,<s 2>)^{l o w}, T=T_{c}}=0, \\
& \left.\frac{\partial V_{e f f}}{\partial s_{2}}\right|_{(<h>,<s 1>,<s 2>)^{h i g h}, T=T_{c}}=0, \\
& \left.\frac{\partial V_{e f f}}{\partial s_{2}}\right|_{(<h>,<s 1>,<s 2>)^{l o w}, T=T_{c}}=0, \\
& \left.V_{e f f}\right|_{(<h>,<s 1>,<s 2>)^{h i g h}, T=T_{c}}=0 \\
& =\left.V_{e f f}\right|_{(<h>,<s 1>,<s 2>)^{l o w}, T=T_{c}} \cdot
\end{aligned}
$$

The last condition guarantees degeneracy and the others guarantee existence of the high and low vacua. There is no analytical solution for the problem, so the calculations are implemented with the CosmoTransitions package [93]. The benchmark points and the corresponding results are presented in Tables 1 and 2, respectively. Here, the exact calculations are performed by CosmoTransitions to get the effective potential, compared to Ref. [87] which used the high temperature expansion. Though, the results of Ref. [87] should be improved for the high temperature expansion case. An extension of the SM with two new scalars was recently studied in Ref. [94], but there are some differences between it and the present work. First, the high temperature expansion was used in [94]. Second, the cubic term $S_{1}^{3}$, which plays a crucial role in the EWPT as a barrier at tree-level, was not considered in [94].

\section{Gravitational waves}

The SFOEWPT may justify not only the BAU but also the GW signal produced by the EWPT. Actually, the EWPT occurs at a temperature lower than $T_{c}$, in which the first broken phase bubbles nucleate in the symmetric phase plasma of the early universe. The transition probability is given by $\Gamma(T)=\Gamma_{0}(T) e^{-S(T)}$ where $\Gamma_{0}(T)$ is of order $\mathcal{O}\left(T^{4}\right)$ and $\mathrm{S}$ is the 4-dimensional action of the critical bubbles. For temperatures sufficiently greater than zero, it can be assumed $S=\frac{S_{3}}{T}$ where the 3-dimensional action is given by 
Table 1 Benchmark points which provide the SFOEWPT

\begin{tabular}{|c|c|c|c|c|c|c|c|c|c|c|}
\hline & $M_{1}(\mathrm{GeV})$ & $M_{2}(\mathrm{GeV})$ & $\theta$ & $\chi(\mathrm{GeV})$ & $\kappa_{1}$ & $\kappa_{3}$ & $\kappa_{4}$ & $\kappa_{5}$ & $\lambda_{2}$ & $Q(\mathrm{GeV})$ \\
\hline BM1 & 25.27 & 655.22 & -9.80 & 115 & -40.72 & 0.0528 & -4.04 & -11.68 & 0.55 & 149 \\
\hline BM2 & 65.74 & 337 & 17.16 & 65.75 & -8.24 & 0.0241 & -34 & 21.44 & 0.79 & 109 \\
\hline BM3 & 83.16 & 235.91 & -18.68 & 69.89 & -40.53 & 0.0132 & 13.48 & -15.44 & 3.62 & 160 \\
\hline BM4 & 195.89 & 434.2 & -20.42 & 96.03 & -55.38 & 0.0322 & -50.92 & 47.82 & 2.36 & 106.7 \\
\hline BM5 & 226.06 & 126.33 & -19.20 & 54.07 & -29.63 & 0.0016 & 7.05 & 5.92 & 1.79 & 104.7 \\
\hline BM6 & 254.18 & 420 & -15.94 & 43.82 & -35.19 & 0.0241 & -13.01 & 48.3 & 1.74 & 91.19 \\
\hline BM7 & 262.86 & 600 & -21.9 & 53.04 & -38.4 & 0.0618 & -2.07 & 73.55 & 3.05 & 91.18 \\
\hline BM8 & 305 & 325 & -6 & 36 & -47 & 0.0012 & -2 & -26.4 & 0.13 & 91.19 \\
\hline
\end{tabular}

Table 2 The values of the VeV of the high and the low phases, $T_{c}$ and the strength of the SFOEWPT

\begin{tabular}{lllrl}
\hline & $\mathrm{VeV}_{\mathrm{c}}^{\text {high }}(\mathrm{GeV})$ & $\mathrm{VeV}_{\mathrm{c}}^{\text {low }}(\mathrm{GeV})$ & $\mathrm{T}_{\mathrm{c}}(\mathrm{GeV})$ & $\xi$ \\
\hline BM1 & $(0,6.66,0)$ & $(152.44,58.12,0)$ & 92.61 & 1.65 \\
BM2 & $(0,212.26,0)$ & $(239.05,67.24,0)$ & 60.33 & 3.96 \\
BM3 & $(0,2.13,0)$ & $(117.2,27.16,0)$ & 115.44 & 1.01 \\
BM4 & $(0,191.74,0)$ & $(214.86,100.1,0)$ & 97.06 & 2.21 \\
BM5 & $(0,110.24,0)$ & $(164.43,76.63,0)$ & 114.18 & 1.44 \\
BM6 & $(0,102.36,0)$ & $(215.56,45.91,0)$ & 97.22 & 2.22 \\
BM7 & $(0,113.79,0)$ & $(222.88,48.07,0)$ & 91.84 & 2.43 \\
BM8 & $(0,72.31,0)$ & $(145.52,48.35,0)$ & 118.13 & 1.23 \\
\hline
\end{tabular}

$S_{3}=4 \pi \int d r r^{2}\left[\frac{1}{2}\left(\partial_{r} \vec{\phi}\right)^{2}+V_{e f f}\right]$.

Here, $\vec{\phi}=(h, s 1, s 2)$. The critical bubble profiles, which minimize the action (4.1), can be calculated from the equation of motions. The temperature for a particular configuration, which gives the nucleation probability of order $\mathcal{O}(1)$, is the nucleation temperature $T_{n}$.

The GW may be produced by the collision of the bubbles at some temperature $T_{*}$, it is usually assumed $T_{*}=T_{n}$. Supposing that the friction force is not enough to prevent the bubbles from running away, the GW signal is given by

$\Omega_{G W} h^{2} \simeq \Omega_{c o l} h^{2}+\Omega_{s w} h^{2}+\Omega_{t u r b} h^{2}$.

As seen, the GW signal is given by the sum of bubbles collision, sound wave and turbulence in the plasma which respectively read $[53,55,56,59,64,73,95,96]$

$$
\begin{aligned}
& \Omega_{c o l} h^{2}= 1.67 \times 10^{-5}\left(\frac{\beta}{H}\right)^{-2} \frac{0.11 v_{b}^{3}}{0.42+v_{b}^{2}} \\
& \times\left(\frac{\kappa \alpha}{1+\alpha}\right)^{2}\left(\frac{g_{*}}{100}\right)^{-\frac{1}{3}} \frac{3.8\left(\frac{f}{f_{c o l}}\right)^{2.8}}{1+2.8\left(\frac{f}{f_{c o l}}\right)^{3.8}},
\end{aligned}
$$

$$
\begin{aligned}
\Omega_{\text {sw }} h^{2}= & 2.65 \\
\times & \times 10^{-6}\left(\frac{\beta}{H}\right)^{-1} v_{b}\left(\frac{\kappa_{v} \alpha}{1+\alpha}\right)^{2} \\
& \times\left(\frac{g_{*}}{100}\right)^{-\frac{1}{3}}\left(\frac{f}{f_{\text {sw }}}\right)^{3}\left(\frac{7}{4+3\left(\frac{f}{f_{\text {sw }}}\right)^{2}}\right)^{\frac{7}{2}}, \\
\Omega_{\text {turb }} h^{2}= & 3.35 \times 10^{-4}\left(\frac{\beta}{H}\right)^{-1} v_{b}\left(\frac{\epsilon \kappa_{v} \alpha}{1+\alpha}\right)^{\frac{3}{2}} \\
& \times\left(\frac{g_{*}}{100}\right)^{-\frac{1}{3}} \frac{\left(\frac{f}{f_{\text {turb }}}\right)^{3}\left(1+\frac{f}{f_{\text {turb }}}\right)^{-\frac{11}{3}}}{1+\frac{8 \pi f}{h_{*}}},
\end{aligned}
$$

with

$$
\begin{aligned}
f_{\text {col }}= & 16.5 \times 10^{-6} \mathrm{~Hz}\left(\frac{0.62}{v_{b}^{2}-0.1 v_{b}+1.8}\right) \\
& \times\left(\frac{\beta}{H}\right)\left(\frac{T_{n}}{100 \mathrm{GeV}}\right)\left(\frac{g_{*}}{100}\right)^{\frac{1}{6}}, \\
f_{\text {sw }}= & 1.9 \times 10^{-5} \mathrm{~Hz}\left(\frac{1}{v_{b}}\right)\left(\frac{\beta}{H}\right)\left(\frac{T_{n}}{100 \mathrm{GeV}}\right)\left(\frac{g_{*}}{100}\right)^{\frac{1}{6}}, \\
f_{\text {turb }}= & 2.7 \times 10^{-5} \mathrm{~Hz}\left(\frac{1}{v_{b}}\right)\left(\frac{\beta}{H}\right)\left(\frac{T_{n}}{100 \mathrm{GeV}}\right)\left(\frac{g_{*}}{100}\right)^{\frac{1}{6}}, \\
h_{*}= & 16.5 \times 10^{-6} \mathrm{~Hz}\left(\frac{T_{n}}{100 \mathrm{GeV}}\right)\left(\frac{g_{*}}{100}\right)^{\frac{1}{6}}, \\
\kappa=1 & -\frac{\alpha_{\infty}}{\alpha}, \\
\kappa_{v}= & \frac{\alpha_{\infty}}{\alpha}\left(\frac{\alpha_{\infty}}{0.73+0.083 \sqrt{\alpha_{\infty}}+\alpha_{\infty}}\right), \\
\alpha_{\infty}= & \frac{30}{24 \pi^{2} g_{*}}\left(\frac{v_{n}}{T_{n}}\right)^{2}\left(6\left(\frac{M_{W}}{v}\right)^{2}\right. \\
& \left.+3\left(\frac{M_{Z}}{v}\right)^{2}+6\left(\frac{M_{\text {top }}}{v}\right)^{2}\right) .
\end{aligned}
$$

$v_{n}$ and $g_{*}$ are the Higgs $\mathrm{VeV}$ and the number of the relativistic degrees of freedom at $T_{n}$, respectively. Here, $\epsilon=0.1$, and $g_{*}$ 
Table 3 The values of the $\mathrm{VeV}$ of the high and the low phases, $T_{n}, \alpha$ and $\beta / H$

\begin{tabular}{lllllr}
\hline & $\mathrm{VeV}_{\mathrm{n}}^{\text {high }}(\mathrm{GeV})$ & $\mathrm{VeV}_{\mathrm{n}}^{\text {low }}(\mathrm{GeV})$ & $\mathrm{T}_{\mathrm{n}}(\mathrm{GeV})$ & $\alpha$ & \multicolumn{1}{c}{$\beta / H$} \\
\hline BM1 & $(0,6.46,0)$ & $(169.49,68.13,0)$ & 89.15 & 0.0324 & 6291.32 \\
BM2 & $(0,212.75,0)$ & $(244.78,63.20,0)$ & 41.61 & 0.2595 & 18459.13 \\
BM3 & $(0,2.09,0)$ & $(127.05,30.59,0)$ & 114.43 & 0.0119 & 27039.55 \\
BM4 & $(0,194.67,0)$ & $(243.13,93.35,0)$ & 51.83 & 0.3131 & 130.43 \\
BM5 & $(0,110.54,0)$ & $(185.04,69.54,0)$ & 110.97 & 0.0245 & 5644.92 \\
BM6 & $(0,103.24,0)$ & $(234.55,41.18,0)$ & 77.05 & 0.0890 & 433.45 \\
BM7 & $(0,114.91,0)$ & $(243.12,42.47,0)$ & 43 & 0.5388 & 150.54 \\
BM8 & $(0,72.67,0)$ & $(157.93,45.79,0)$ & 115.38 & 0.0175 & 9306.63 \\
\hline
\end{tabular}

is read from the MicrOMEGAs package [97,98]. Still, there are three important parameters which should be defined. One of them is the bubble wall velocity, since assumed that the bubbles run away, given by $v_{b} \simeq 1$. The two others, $\alpha$ and $\beta$, are given as follows

$$
\begin{aligned}
& \alpha=\left.\frac{\rho_{v a c}}{\rho^{*}}\right|_{T_{n}}, \\
& \beta=\left.\left[H T \frac{d}{d T}\left(\frac{S_{3}}{T}\right)\right]\right|_{T_{n}},
\end{aligned}
$$

where $\quad \rho_{\text {vac }}=\left(V_{\text {eff }}^{\text {high }}-T d V_{\text {eff }}^{\text {high }} / d T\right)-$ $\left(V_{\text {eff }}^{\text {low }}-T d V_{\text {eff }}^{\text {low }} / d T\right), \rho^{*}=g_{*} \pi^{2} T_{n}^{4} / 30$ and $H_{n}$ are the latent heat (vacuum energy density) released by the EWPT, the background energy density of the plasma and Hubble parameter at $T_{n}$, respectively. Using the CosmoTransitions package [93], the parameters $\alpha, \beta / H, v_{n}$ and $T_{n}$ are calculated and presented in Table 3. In Fig. 1, the GW signals are plotted versus frequency for the benchmark points of Table 1 . To check if the GW signals for the benchmark points 1 fall within the sensitivity of GW detectors, the sensitivity curves of eLISA, ALIA, DECIGO and BBO detectors ${ }^{4}$ are also plotted in the Fig. 1. As seen from the Fig. 1, the dashed blue line corresponding to the GW signal for the BM7 may be detected by N2A1M5L6 and N2A5M5L6 configurations of eLISA and BBO detectors. The dashed red and yellow lines corresponding, respectively, to the GW signal for the BM4 and the BM6 may be detected by N2A5M5L6 configuration of eLISA and BBO detectors. The dashed orange line corresponding to the BM2 may be detected by DECIGO and BBO detectors. The dashed green, cyan and purple lines corresponding, respectively, to the GW signal for BM1, BM5 and BM 8 cannot be detected by the mentioned detectors. The GW signal for the BM3 is not big enough to be shown at the scale of the Fig. 1.

\footnotetext{
${ }_{4}$ The sensitivity curves of four representative configurations of eLISA are taken from [73]. The ALIA, the DECIGO and the BBO sensitivity curves are taken from GWPLOTTER. The reader is referred to Ref. [99] for details.
}

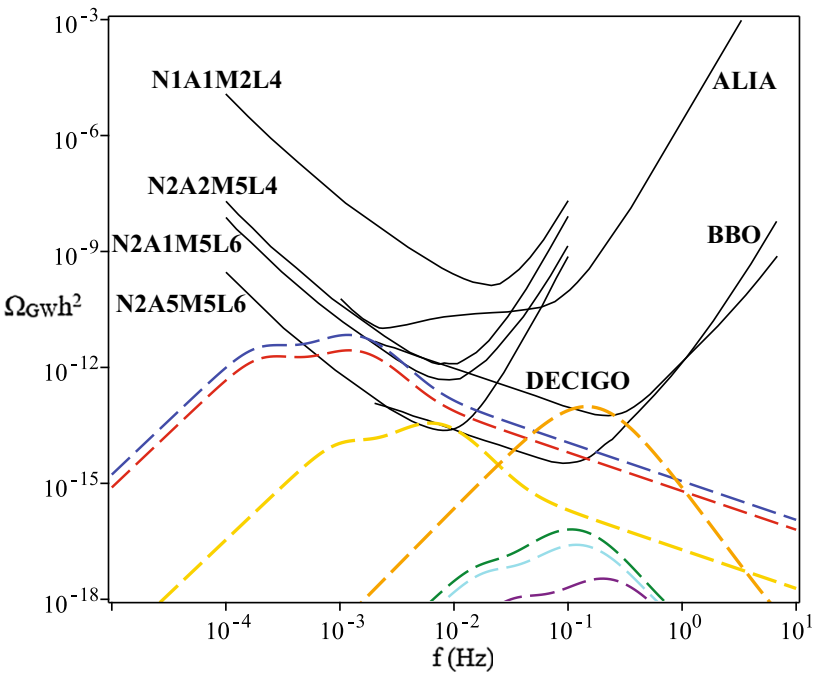

Fig. 1 The dashed blue, red, yellow, orange, green, cyan and purple lines represent the GW signal for BM7, BM4, BM6, BM2, BM1, BM5 and BM8, respectively. The solid black lines represent the sensitivity curves and are labeled by the name of the detectors. For eLISA, the sensitivity curves are labeled by the name of the configuration

According to the Tables 2 and 3 , it seems that $\mathrm{BM} 2$ is a special point. The value of $\beta / H$ is large at this point, while, the nucleation temperature is not very close to the critical temperature. At the same time, $T_{n}$ is low and $\alpha$ is large. ${ }^{5}$ To clarify the situation of BM2, the phase transition properties of BM2 are shown in Fig. 2. As seen from the Fig. 2a, the slope of $S_{3} / T$ increases around $T_{n}$ which indicates the parameter $\beta / H$ is large. The physics of this situation can be described by the tunneling profile, the norm of phases as a function of temperature, and the contour levels of the potential with the tunneling path. As seen from Fig. 2b, d, the center of bubble is far away from the stable vacuum. Also from Fig. 2c, it is seen that the transition occurs at a temperature where the unstable vacuum is close to disappearance. The values of potential at high and low phases are, respectively, $V_{\text {eff }}^{\text {high }}=-91583128.19\left(\mathrm{GeV}^{4}\right)$

\footnotetext{
5 The authors thank an anonymous referee for pointing this out.
} 


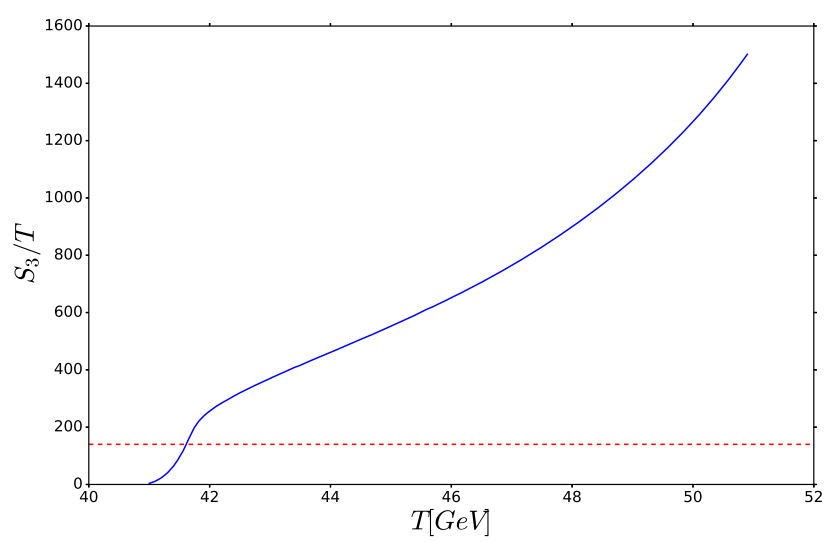

(a)

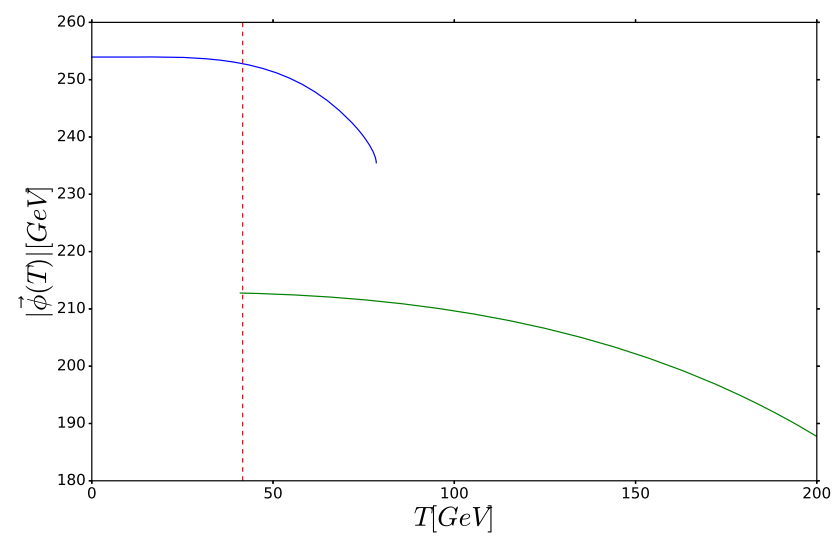

(c)

Fig. 2 Phase transition properties of BM2: the subfigure a presents $S_{3} / T$ versus temperature, the dashed horizontal red line shows $S_{3} / T=$ 140 where nucleation occurs. The subfigure $\mathbf{b}$ presents the tunneling profile as a function of radius. The subfigure $\mathbf{c}$ presents the norms of high (green line) and low (blue line) phases as functions of tempera-

and $V_{e f f}^{\text {low }}=-101840540.47\left(\mathrm{GeV}^{4}\right)$, which give the pressure difference $\Delta p=10257412.28\left(\mathrm{GeV}^{4}\right)$. The barrier location is at $\left(h, s_{1}, s_{2}\right)=(19.84,212.19,0)$ with $V_{\text {eff }}=-91582001.71\left(\mathrm{GeV}^{4}\right)$ which gives the barrier height $\Delta V_{\text {barrier height }}=1126.48\left(\mathrm{GeV}^{4}\right)$. Clearly, the barrier height is very small, $\Delta V_{\text {barrier height }} / \Delta p=0.0001$. Due to the reasons given above, the bubbles are extremely thick walled. Since the barrier height is very small, the transition duration is very short, accordingly, the parameter $\beta / H$ is quite large. This extremely thick walled case is similar to the second-order phase transition in which $\beta / H \rightarrow \infty$ and there is no barrier. Moreover, there is another interesting note for BM2. Due to the cubic term $s_{1}^{3}$, it is expected that the model has a sizable barrier at tree-level like the supercooled scenario discussed in [78], but this is not the case for BM2. At this point, the model mimics the behavior of

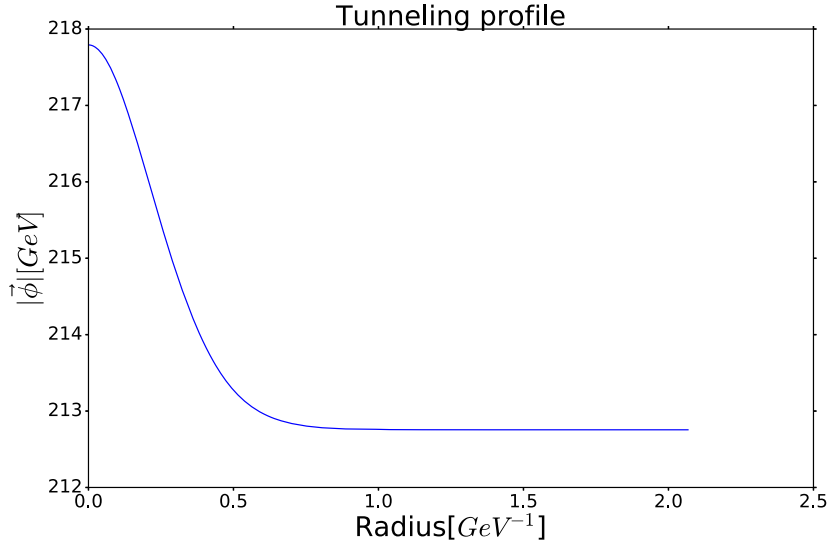

(b)

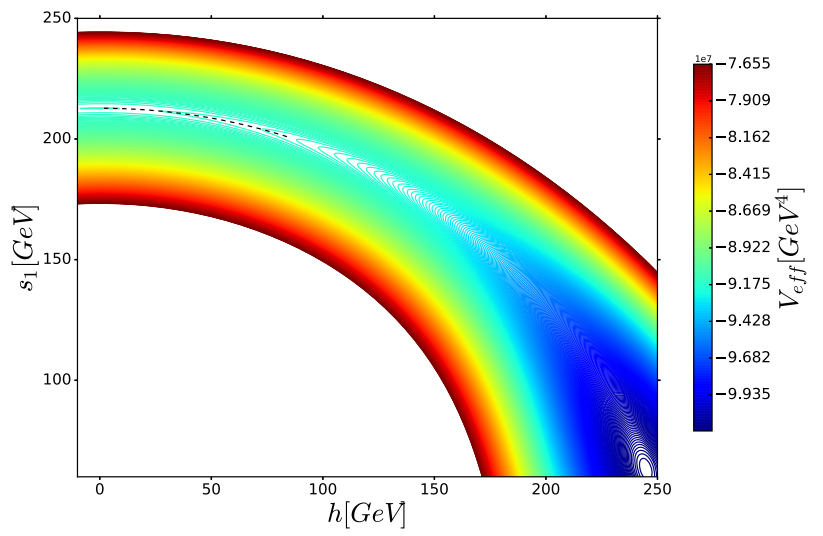

(d)

ture, the dashed vertical red line shows the nucleation temperature. The subfigure $\mathbf{d}$ presents the contour levels of the potential at the nucleation temperature $T_{n}=41.61(\mathrm{GeV})$, the dashed black line shows the tunneling path

supercooled phase transitions with the supercooling parame$\operatorname{ter}\left(T_{c}-T_{n}\right) / T_{c}=0.31$, though, the transition is short-lived.

\section{Dark matter}

As mentioned prior, imposing the $Z_{2}$ symmetry on $S_{2}$ makes it a viable candidate for the DM. Considering the freeze-out formalism, the DM relic density abundance can be calculated by solving the Boltzmann equation,

$\frac{d n}{d t}=-3 H n-<\sigma v>\left(n^{2}-n_{e q}^{2}\right)$,

where $\mathrm{n}, \mathrm{H},\langle\sigma v>$ are the number of the DM particles, the Hubble parameter and the thermally-averaged cross section for the DM annihilation, respectively. It is customary to rewrite the Boltzmann equation in terms of $Y=n / s$, where $s$ 
Table 4 The values of the DM relic abundance and the spinindependent cross sections

\begin{tabular}{llll}
\hline & $\Omega_{D M} h^{2}$ & $\sigma_{S I}^{\text {proton }}(\mathrm{pb})$ & $\sigma_{S I}^{\text {neutron }}(\mathrm{pb})$ \\
\hline BM1 & 0.104 & $5.151 \times 10^{-12}$ & $5.315 \times 10^{-12}$ \\
BM2 & 0.12 & $6.469 \times 10^{-12}$ & $6.667 \times 10^{-12}$ \\
BM3 & $6.28 \times 10^{-2}$ & $9.447 \times 10^{-12}$ & $9.729 \times 10^{-12}$ \\
BM4 & 0.109 & $5.038 \times 10^{-11}$ & $5.195 \times 10^{-11}$ \\
BM5 & 0.108 & $6.186 \times 10^{-12}$ & $6.363 \times 10^{-12}$ \\
BM6 & 0.12 & $4.223 \times 10^{-12}$ & $4.354 \times 10^{-12}$ \\
BM7 & $5.90 \times 10^{-2}$ & $4.755 \times 10^{-11}$ & $4.906 \times 10^{-11}$ \\
BM8 & 0.12 & $2.480 \times 10^{-11}$ & $2.556 \times 10^{-11}$ \\
\hline
\end{tabular}

is the total entropy density of the universe, the result is [100]

$$
\begin{aligned}
\frac{d Y}{d x} & =-\left(\frac{45 G g_{*}}{\pi}\right)^{-\frac{1}{2}} \frac{M h_{*}}{x^{2}}\left(1+\frac{1}{3} \frac{T}{h_{*}} \frac{d h_{*}}{d T}\right) \\
& <\sigma v>\left(Y^{2}-Y_{e q}^{2}\right),
\end{aligned}
$$

where $x=M / T, M$ is the DM mass. $h_{*}$ is the effective degree of freedom for the entropy densities. The DM relic density abundance reads,

$\Omega_{D M} h^{2} \simeq(2.79 \pm 0.05) \times 10^{8}\left(\frac{M}{\mathrm{GeV}}\right) Y(0)$.

It is assumed the usual SM particles only interact with Higgs in this model, so the annihilation channels for DM via the Higgs portal s-channel are $s_{2} s_{2} \rightarrow W^{+} W^{-}, Z Z, f \bar{f}$. Also, there exists $s_{2} s_{2} \rightarrow \phi_{i} \phi_{j}\left(\right.$ with $\phi_{i(j)}=h, s_{1}$ and $\left.i(j)=1,2\right)$ via $s, t$ and $u$ channels and four-point interactions.

The parameter space is constrained by the direct detection DM searches. To do this, one needs to calculate the spinindependent cross section for DM-nucleon scattering, ${ }^{6}$ and compares the result with the XENON1T 2018 experiment data [101]. The spin-independent cross section is given by

$\sigma_{S I}=\frac{4 M_{s_{2}}^{2} M_{N}^{2}}{\pi\left(M_{S 2}+M_{N}\right)^{2}}\left|\mathcal{M}_{s_{2}-N}\right|^{2}$,

where $M_{s_{2}}, M_{N}$ and $\mathcal{M}_{s_{2}-N}$ are the DM mass, the nucleus mass and the scattering amplitude at low energy limit, respectively. $\mathcal{M}_{s_{2}-N}$ is related to $\mathcal{M}_{s_{2}-\text { quark }}$, so, calculating effective Lagrangian coefficients and nucleon form factors, $\mathcal{M}_{s_{2}-N}$ can be obtained from $\mathcal{M}_{s_{2}-\text { quark }}$. Here, the model is implemented in SARAH [102-104], the model spectrum is obtained by SPheno $[105,106]$ and the DM properties are studied by MicrOMEGAs $[97,98]$. The results are presented in Table 4.

\footnotetext{
6 The spin-dependent case is not studied here, because the DM candidate is assumed to be a scalar.
}

As seen in Table 4, the relic density of all benchmark points is compatible with Planck 2018 data which reports $\Omega_{c} h^{2}=0.120 \pm 0.001 .^{7}$ Moreover, the results fit with XENON1T 2018 experiment which gives an upper limit, less than LUX 2017 [107] and PandaX-II 2017 [108] reports, on the DM-nucleon spin-independent elastic scattering cross section. In the DM study, there are two differences with Ref. [94]. The first is the $s_{1}^{3}$ interaction which gives a significant contribution to the DM annihilation through $s_{1} \mathrm{~s}$ channel, and consequently to relic density. The second is the spin-independent cross section which was taken to be zero in Ref. [94], but the more realistic case like here is to have a non-zero DM-nucleon cross section, if weakly interacting massive particles (WIMP) constitute the DM. This is the main idea behind LUX, PandaX-II and XENON1T experiments.

\section{Conclusions}

The main goal of this work has been to investigate the EWPT, the GW and the DM issues in an extension of the SM by adding two scalar degrees of freedom. To reach the goal, it has been assumed that one of the new scalars has a nonzero $\mathrm{VeV}$ to assist the phase transition and the other has no $\mathrm{VeV}$ to be a viable DM candidate. It has been seen if one takes the most general renormalizable form of the potential, the model can represent all the signals together. As seen from Tables 2 and 3, the model can have phase transitions from strong $(\xi \sim 1)$ to very strong $(\xi \sim 4)$. From Fig. 1 , the model presents the GW signals from the frequency range of $10^{-5}(\mathrm{~Hz})$ to $10(\mathrm{~Hz})$ which are detectable by eLISA, BBO and DECIGO. From Table 4, the model provides the DM signals which are in agreement with the Planck 2018 data and the XENON1T 2018 experiment. It is seen that the DM candidate may be quite massive with a mass greater than $100(\mathrm{GeV})$ which belongs to the extremely cold DM; although, since the model has a rich parameter space, the lighter DMs might be found by performing a Monte Carlo simulation via a computer cluster. With all of these, it can be concluded that the SFOEWPT, the GW and the DM signals can successfully be described by the present model as an extension of the SM with two additional real gauge singlet scalars. As a final note, it has been assumed that the GW production from bubble collisions follows from thinwall and envelope approximations which is usual in the literature. In this assumption, only uncollided parts of the bubbles are taken into account as the GW sources. Recently, it has been shown that the GW production from bubble collisions is analytically solvable $[109,110]$. Also, the possibility of using GWs and collider experiments to constrain the EWPT has been discussed in [111]. It is left for future work to study

\footnotetext{
$\overline{70.05 \leq \Omega_{c} h^{2}} \leq 0.12$ would be acceptable.
} 
the GW signals of the present model using these recent studies.

Acknowledgements The authors would like to thank Ryusuke Jinno for comments on the GW production from bubble collisions.

Data Availability Statement This manuscript has no associated data or the data will not be deposited. [Authors' comment: All the experimental data and the codes used for this publication are publicly available, as referenced in the text.]

Open Access This article is distributed under the terms of the Creative Commons Attribution 4.0 International License (http://creativecomm ons.org/licenses/by/4.0/), which permits unrestricted use, distribution, and reproduction in any medium, provided you give appropriate credit to the original author(s) and the source, provide a link to the Creative Commons license, and indicate if changes were made.

Funded by SCOAP ${ }^{3}$.

\section{References}

1. ATLAS Collaboration, G. Aad et al., Observation of a new particle in the search for the Standard Model Higgs boson with the ATLAS detector at the LHC. Phys. Lett. B 716, 1-29 (2012). arXiv: 1207.7214

2. C.M.S. Collaboration, S. Chatrchyan et al., Observation of a new boson at a mass of $125 \mathrm{GeV}$ with the CMS experiment at the LHC. Phys. Lett. B 716, 30-61 (2012). arXiv:1207.7235

3. J.M. Cline, K. Kainulainen, A New source for electroweak baryogenesis in the MSSM. Phys. Rev. Lett. 85, 5519-5522 (2000). arXiv:hep-ph/0002272

4. S.J. Huber, T. Konstandin, T. Prokopec, M.G. Schmidt, Electroweak phase transition and baryogenesis in the nMSSM. Nucl. Phys. B 757, 172-196 (2006). arXiv:hep-ph/0606298

5. S.J. Huber, T. Konstandin, T. Prokopec, M.G. Schmidt, Baryogenesis in the MSSM, nMSSM and NMSSM. Nucl. Phys. A 785, 206-209 (2007). arXiv:hep-ph/0608017

6. M. Pietroni, The electroweak phase transition in a nonminimal supersymmetric model. Nucl. Phys. B 402, 27-45 (1993). arXiv:hep-ph/9207227

7. A.T. Davies, C.D. Froggatt, R.G. Moorhouse, Electroweak baryogenesis in the next-to-minimal supersymmetric model. Phys. Lett. B 372, 88-94 (1996). arXiv:hep-ph/9603388

8. S .W. Ham, S .K. OH, C .M. Kim, E .J. Yoo, D. Son, Electroweak phase transition in a nonminimal supersymmetric model. Phys. Rev. D 70, 075001 (2004). arXiv:hep-ph/0406062

9. A. Menon, D.E. Morrissey, Higgs Boson signatures of MSSM electroweak baryogenesis. Phys. Rev. D 79, 115020 (2009). arXiv:0903.3038

10. M. Carena, G. Nardini, M. Quiros, C.E.M. Wagner, MSSM electroweak baryogenesis and LHC data. JHEP 02, 001 (2013). arXiv: 1207.6330

11. S.J. Huber, T. Konstandin, Production of gravitational waves in the nMSSM. JCAP 0805, 017 (2008). arXiv:0709.2091

12. J. Kozaczuk, S. Profumo, L.S. Haskins, C.L. Wainwright, Cosmological phase transitions and their properties in the NMSSM. JHEP 01, 144 (2015). arXiv:1407.4134

13. J. Kozaczuk, S. Profumo, M.J. Ramsey-Musolf, C.L. Wainwright, Supersymmetric electroweak baryogenesis via resonant sfermion sources. Phys. Rev. D 86, 096001 (2012). arXiv: 1206.4100

14. G. Jungman, M. Kamionkowski, K. Griest, Supersymmetric dark matter. Phys. Rept. 267, 195-373 (1996). arXiv:hep-ph/9506380
15. A. Menon, D.E. Morrissey, C.E.M. Wagner, Electroweak baryogenesis and dark matter in the nMSSM. Phys. Rev. D 70, 035005 (2004). arXiv:hep-ph/0404184

16. V. Cirigliano, S. Profumo, M.J. Ramsey-Musolf, Baryogenesis, electric dipole moments and dark matter in the MSSM. JHEP 07, 002 (2006). arXiv:hep-ph/0603246

17. J.-J. Cao, K.-I. Hikasa, W. Wang, J.M. Yang, K.-I. Hikasa, W.-Y. Wang, J.M. Yang, Light dark matter in NMSSM and implication on Higgs phenomenology. Phys. Lett. B 703, 292-297 (2011). arXiv: 1104.1754

18. P.H. Damgaard, A. Haarr, D. O'Connell, A. Tranberg, Effective field theory and electroweak baryogenesis in the singlet-extended standard model. JHEP 02, 107 (2016). arXiv:1512.01963

19. V. Vaskonen, Electroweak baryogenesis and gravitational waves from a real scalar singlet. Phys. Rev. D 95(12), 123515 (2017). arXiv: 1611.02073

20. A. Beniwal, M. Lewicki, J.D. Wells, M. White, A.G. Williams, Gravitational wave, collider and dark matter signals from a scalar singlet electroweak baryogenesis. JHEP 08, 108 (2017). arXiv: 1702.06124

21. C.-Y. Chen, J. Kozaczuk, I.M. Lewis, Non-resonant collider signatures of a singlet-driven electroweak phase transition. JHEP $\mathbf{0 8}$, 096 (2017). arXiv: 1704.05844

22. J.R. Espinosa, T. Konstandin, F. Riva, Strong electroweak phase transitions in the standard model with a singlet. Nucl. Phys. B 854, 592-630 (2012). arXiv:1107.5441

23. J.M. Cline, P.-A. Lemieux, Electroweak phase transition in two Higgs doublet models. Phys. Rev. D 55, 3873-3881 (1997). arXiv:hep-ph/9609240

24. L. Fromme, S.J. Huber, M. Seniuch, Baryogenesis in the two-Higgs doublet model. JHEP 11, 038 (2006). arXiv:hep-ph/0605242

25. Z. Kang, P. Ko, T. Matsui, Strong first order EWPT and strong gravitational waves in $\mathrm{Z}_{3}$-symmetric singlet scalar extension. JHEP 02, 115 (2018). arXiv:1706.09721

26. G.C. Dorsch, S.J. Huber, J.M. No, A strong electroweak phase transition in the 2HDM after LHC8. JHEP 10, 029 (2013). arXiv: 1305.6610

27. A. Haarr, A. Kvellestad, T.C. Petersen, Disfavouring Electroweak Baryogenesis and a Hidden Higgs in a CP-Violating Two-HiggsDoublet Model. arXiv:1611.05757

28. J.F. Gunion, R. Vega, J. Wudka, Higgs triplets in the standard model. Phys. Rev. D 42, 1673-1691 (1990)

29. P. Fileviez Perez, H .H. Patel, M. Ramsey-Musolf, K. Wang, Triplet scalars and dark matter at the LHC. Phys. Rev. D 79, 055024 (2009). arXiv:0811.3957

30. T. Alanne, K. Tuominen, V. Vaskonen, Strong phase transition, dark matter and vacuum stability from simple hidden sectors. Nucl. Phys. B 889, 692-711 (2014). arXiv:1407.0688

31. H.H. Patel, M.J. Ramsey-Musolf, Baryon washout, electroweak phase transition, and perturbation theory. JHEP 07, 029 (2011). arXiv: 1101.4665

32. V.A. Kuzmin, V.A. Rubakov, M.E. Shaposhnikov, On the anomalous electroweak Baryon number nonconservation in the early universe. Phys. Lett. 155B, 36 (1985)

33. M.E. Shaposhnikov, Baryon asymmetry of the universe in standard electroweak theory. Nucl. Phys. B 287, 757-775 (1987)

34. M. Dine, A. Kusenko, The origin of the matter-antimatter asymmetry. Rev. Mod. Phys. 76, 1 (2003). arXiv:hep-ph/0303065

35. J. M. Cline, Baryogenesis, in Les Houches Summer SchoolSession 86: Particle Physics and Cosmology: The Fabric of Spacetime Les Houches, France, July 31-August 25, 2006 (2006). arXiv:hep-ph/0609145

36. L. Canetti, M. Drewes, M. Shaposhnikov, Matter and Antimatter in the Universe. New J. Phys. 14, 095012 (2012). arXiv:1204.4186 
37. D. Land, E.D. Carlson, Two stage phase transition in two Higgs models. Phys. Lett. B 292, 107-112 (1992). arXiv:hep-ph/9208227

38. A. Hammerschmitt, J. Kripfganz, M.G. Schmidt, Baryon asymmetry from a two stage electroweak phase transition? Z. Phys. C 64, 105-110 (1994). arXiv:hep-ph/9404272

39. H.H. Patel, M.J. Ramsey-Musolf, Stepping into electroweak symmetry breaking: phase transitions and higgs phenomenology. Phys. Rev. D 88, 035013 (2013). arXiv:1212.5652

40. W. Huang, Z. Kang, J. Shu, P. Wu, J.M. Yang, New insights in the electroweak phase transition in the NMSSM. Phys. Rev. D 91(2), 025006 (2015). arXiv:1405.1152

41. N. Blinov, J. Kozaczuk, D.E. Morrissey, C. Tamarit, Electroweak baryogenesis from exotic electroweak symmetry breaking. Phys. Rev. D 92(3), 035012 (2015). arXiv:1504.05195

42. J. Ellis, M. Lewicki, J.M. No, On the maximal strength of a firstorder electroweak phase transition and its gravitational wave signal. Submitted to: JCAP (2018) arXiv: 1809.08242

43. M.J. Baker, M. Breitbach, J. Kopp, L. Mittnacht, Dynamic freeze-in: impact of thermal masses and cosmological phase transitions on dark matter production. JHEP 03, 114 (2018). arXiv: 1712.03962

44. D. Croon, V. Sanz, G. White, Model discrimination in gravitational wave spectra from dark phase transitions. JHEP 08, 203 (2018). arXiv: 1806.02332

45. A. Beniwal, M. Lewicki, M. White, A.G. Williams, Gravitational Waves and Electroweak Baryogenesis in a Global Study of the Extended Scalar Singlet Model. arXiv:1810.02380

46. F.P. Huang, X. Zhang, Probing the Gauge Symmetry Breaking of the Early Universe in 3-3-1 Models and Beyond by Gravitational Waves. arXiv: 1701.04338

47. K. Hashino, M. Kakizaki, S. Kanemura, P. Ko, T. Matsui, Gravitational waves from first order electroweak phase transition in models with the $\mathrm{U}(1)_{X}$ gauge symmetry. JHEP 06, 088 (2018). arXiv: 1802.02947

48. A. Mazumdar, G. White, Cosmic Phase Transitions: Their Applications and Experimental Signatures. arXiv: 1811.01948

49. P. Ghosh, A.K. Saha, A. Sil, Study of electroweak vacuum stability from extended higgs portal of dark matter and neutrinos. Phys. Rev. D 97(7), 075034 (2018). arXiv:1706.04931

50. A. Kosowsky, M.S. Turner, R. Watkins, Gravitational radiation from colliding vacuum bubbles. Phys. Rev. D 45, 4514-4535 (1992)

51. A. Kosowsky, M.S. Turner, R. Watkins, Gravitational waves from first order cosmological phase transitions. Phys. Rev. Lett. 69, 2026-2029 (1992)

52. A. Kosowsky, M.S. Turner, Gravitational radiation from colliding vacuum bubbles: envelope approximation to many bubble collisions. Phys. Rev. D 47, 4372-4391 (1993). arXiv:astro-ph/9211004

53. M. Kamionkowski, A. Kosowsky, M.S. Turner, Gravitational radiation from first order phase transitions. Phys. Rev. D 49, $2837-$ 2851 (1994). arXiv:astro-ph/9310044

54. C. Caprini, R. Durrer, G. Servant, Gravitational wave generation from bubble collisions in first-order phase transitions: an analytic approach. Phys. Rev. D 77, 124015 (2008). arXiv:0711.2593

55. S.J. Huber, T. Konstandin, Gravitational wave production by collisions: more bubbles. JCAP 0809, 022 (2008). arXiv:0806.1828

56. M. Hindmarsh, S.J. Huber, K. Rummukainen, D.J. Weir, Gravitational waves from the sound of a first order phase transition. Phys. Rev. Lett. 112, 041301 (2014). arXiv:1304.2433

57. J.T. Giblin Jr., J.B. Mertens, Vacuum bubbles in the presence of a relativistic fluid. JHEP 12, 042 (2013). arXiv:1310.2948

58. J.T. Giblin, J.B. Mertens, Gravitional radiation from first-order phase transitions in the presence of a fluid. Phys. Rev. D 90(2), 023532 (2014). arXiv:1405.4005
59. M. Hindmarsh, S.J. Huber, K. Rummukainen, D.J. Weir, Numerical simulations of acoustically generated gravitational waves at a first order phase transition. Phys. Rev. D $92(12), 123009$ (2015). arXiv: 1504.03291

60. C. Caprini, R. Durrer, Gravitational waves from stochastic relativistic sources: primordial turbulence and magnetic fields. Phys. Rev. D 74, 063521 (2006). arXiv:astro-ph/0603476

61. T. Kahniashvili, A. Kosowsky, G. Gogoberidze, Y. Maravin, Detectability of gravitational waves from phase transitions. Phys. Rev. D 78, 043003 (2008). arXiv:0806.0293

62. T. Kahniashvili, L. Campanelli, G. Gogoberidze, Y. Maravin, B. Ratra, Gravitational radiation from primordial helical inverse cascade MHD turbulence. Phys. Rev. D 78, 123006 (2008). arXiv:0809.1899 [Erratum: Phys. Rev. D79,109901(2009)]

63. T. Kahniashvili, L. Kisslinger, T. Stevens, Gravitational radiation generated by magnetic fields in cosmological phase transitions. Phys. Rev. D 81, 023004 (2010). arXiv:0905.0643

64. C. Caprini, R. Durrer, G. Servant, The stochastic gravitational wave background from turbulence and magnetic fields generated by a first-order phase transition. JCAP 0912, 024 (2009). arXiv:0909.0622

65. Virgo, LIGO Scientific Collaboration, B.P. Abbott et al., Observation of gravitational waves from a binary black hole merger. Phys. Rev. Lett.116, 6, 061102 (2016) arXiv:1602.03837

66. Virgo, LIGO Scientific Collaboration, B.P. Abbott et al., GW151226: observation of gravitational waves from a 22-solarmass binary black hole coalescence. Phys. Rev. Lett.116, 24, 241103 (2016) arXiv: 1606.04855

67. Virgo, LIGO Scientific Collaboration, B.P. Abbott et al., First search for gravitational waves from known pulsars with advanced LIGO. Astrophys. J. 839(1), 12 (2017) arXiv:1701.07709]. [Erratum: Astrophys. J.851,no.1,71(2017)

68. VIRGO, LIGO Scientific Collaboration, B.P. Abbott et al., GW170104: Observation of a 50-solar-mass binary black hole coalescence at Redshift 0.2. Phys. Rev. Lett. 118(22), 221101 (2017) arXiv:1706.01812. [Erratum: Phys. Rev. Lett.121,no.12,129901(2018)]

69. Virgo, LIGO Scientific Collaboration, B.P. Abbott et al., GW170814: a three-detector observation of gravitational waves from a binary black hole coalescence. Phys. Rev. Lett. 119(14), 141101 (2017) arXiv: 1709.09660

70. Virgo, LIGO Scientific Collaboration, B. Abbott et al., GW170817: observation of gravitational waves from a binary neutron star inspiral. Phys. Rev. Lett. 119(16), 161101 (2017) arXiv: 1710.05832

71. Virgo, Fermi-GBM, INTEGRAL, LIGO Scientific Collaboration, B.P. Abbott et al., Gravitational waves and gamma-rays from a binary neutron star merger: GW170817 and GRB 170817A. Astrophys. J. 848(2), L13 (2017) arXiv:1710.05834

72. Virgo, LIGO Scientific Collaboration, B.P. Abbott et al., GW170608: observation of a 19-solar-mass binary black hole coalescence. Astrophys. J. 851(2), L35 (2017) arXiv:1711.05578

73. C. Caprini et al., Science with the space-based interferometer eLISA. II: Gravitational waves from cosmological phase transitions. JCAP 1604(04), 001 (2016). arXiv:1512.06239

74. D.J. Weir, Gravitational waves from a first order electroweak phase transition: a brief review. Philos. Trans. R. Soc. Lond. A 376(2114), 20170126 (2018). arXiv:1705.01783

75. C. Caprini, D.G. Figueroa, Cosmological backgrounds of gravitational waves. Class. Quant. Gravit. 35(16), 163001 (2018). arXiv: 1801.04268

76. S.V. Demidov, D.S. Gorbunov, D.V. Kirpichnikov, Gravitational waves from phase transition in split NMSSM. Phys. Lett. B 779, 191-194 (2018). arXiv:1712.00087

77. A. Kobakhidze, A. Manning, J. Yue, Gravitational waves from the phase transition of a nonlinearly realized electroweak gauge 
symmetry. Int. J. Mod. Phys. D 26(10), 1750114 (2017). arXiv: 1607.00883

78. A. Kobakhidze, C. Lagger, A. Manning, J. Yue, Gravitational waves from a supercooled electroweak phase transition and their detection with pulsar timing arrays. Eur. Phys. J. C 77(8), 570 (2017). arXiv: 1703.06552

79. P.S.B. Dev, A. Mazumdar, Probing the scale of new physics by advanced LIGO/VIRGO. Phys. Rev. D 93(10), 104001 (2016). arXiv: 1602.04203

80. Planck Collaboration, N. Aghanim et al., Planck 2018 Results. VI. Cosmological Parameters. arXiv:1807.06209

81. P. Athron, J.M. Cornell, F. Kahlhoefer, J. Mckay, P. Scott, S. Wild, Impact of vacuum stability, perturbativity and XENON1T on global fits of $\mathbb{Z}_{2}$ and $\mathbb{Z}_{3}$ scalar singlet dark matter. Eur. Phys. J. C 78(10), 830 (2018). arXiv:1806.11281

82. N. Bernal, C. Cosme, T. Tenkanen, Phenomenology of SelfInteracting Dark Matter in a Matter-Dominated Universe. arXiv: 1803.08064

83. S. Baum, M. Carena, N.R. Shah, C.E.M. Wagner, Higgs portals for thermal dark matter, EFT perspectives and the NMSSM. JHEP 04, 069 (2018). arXiv:1712.09873

84. T. Li, Revisiting the direct detection of dark matter in simplified models. Phys. Lett. B 782, 497-502 (2018). arXiv:1804.02120

85. P. Bandyopadhyay, E.J. Chun, R. Mandal, Scalar dark matter in leptophilic two-higgs-doublet model. Phys. Lett. B 779, 201-205 (2018). arXiv: 1709.08581

86. J. Yepes, Top Partners Tackling Vector Dark Matter. arXiv: 1811.06059

87. A. Tofighi, O.N. Ghodsi, M. Saeedhoseini, Phase transition in multi-scalar-singlet extensions of the standard model. Phys. Lett. B 748, 208-211 (2015). arXiv:1510.00791

88. ATLAS, CMS Collaboration, G. Aad et al., Combined measurement of the Higgs Boson mass in $p p$ collisions at $\sqrt{s}=7$ and $8 \mathrm{TeV}$ with the ATLAS and CMS experiments. Phys. Rev. Lett. 114, 191803 (2015). arXiv: 1503.07589

89. S. Profumo, M.J. Ramsey-Musolf, C.L. Wainwright, P. Winslow, Singlet-catalyzed electroweak phase transitions and precision Higgs Boson studies. Phys. Rev. D 91(3), 035018 (2015). arXiv: 1407.5342

90. W. Chao, Hiding Scalar Higgs Portal Dark Matter. arXiv:1601.06714

91. M. Quiros, Finite temperature field theory and phase transitions. In: Proceedings, Summer School in High-Energy Physics and Cosmology: Trieste, Italy, June 29-July 17, 1998, pp. 187-259 (1999). arXiv:hep-ph/9901312

92. C. Delaunay, C. Grojean, J.D. Wells, Dynamics of nonrenormalizable electroweak symmetry breaking. JHEP 04, 029 (2008). arXiv:0711.2511

93. C.L. Wainwright, CosmoTransitions: computing cosmological phase transition temperatures and bubble profiles with multiple fields. Comput. Phys. Commun. 183, 2006-2013 (2012). arXiv: 1109.4189

94. W. Chao, H.-K. Guo, J. Shu, Gravitational wave signals of electroweak phase transition triggered by dark matter. JCAP 1709(09), 009 (2017). arXiv: 1702.02698
95. P. Binetruy, A. Bohe, C. Caprini, J.-F. Dufaux, Cosmological backgrounds of gravitational waves and eLISA/NGO: phase transitions, cosmic strings and other sources. JCAP 1206, 027 (2012). arXiv: 1201.0983

96. J.R. Espinosa, T. Konstandin, J.M. No, G. Servant, Energy budget of cosmological first-order phase transitions. JCAP 1006, 028 (2010). arXiv:1004.4187

97. G. Bélanger, F. Boudjema, A. Goudelis, A. Pukhov, B. Zaldivar, micrOMEGAs5.0: freeze-in. Comput. Phys. Commun. 231, 173186 (2018). arXiv: 1801.03509

98. D. Barducci, G. Belanger, J. Bernon, F. Boudjema, J. Da Silva, S. Kraml, U. Laa, A. Pukhov, Collider limits on new physics within micrOMEGAs_4.3. Comput. Phys. Commun. 222, 327338 (2018). arXiv: 1606.03834

99. C.J. Moore, R.H. Cole, C.P.L. Berry, Gravitational-wave sensitivity curves. Class. Quant. Gravit. 32(1), 015014 (2015). arXiv: 1408.0740

100. P. Gondolo, G. Gelmini, Cosmic abundances of stable particles: improved analysis. Nucl. Phys. B 360, 145-179 (1991)

101. XENON Collaboration, E. Aprile et al., Dark matter search results from a one ton-year exposure of XENON1T. Phys. Rev. Lett. 121(11), 111302 (2018) arXiv:1805.12562

102. F. Staub,SARAH. arXiv:0806.0538

103. F. Staub, SARAH 4: a tool for (not only SUSY) model builders. Comput. Phys. Commun. 185, 1773-1790 (2014). arXiv: 1309.7223

104. F. Staub, From superpotential to model files for FeynArts and CalcHep/CompHep. Comput. Phys. Commun. 181, 1077-1086 (2010). arXiv:0909.2863

105. W. Porod, F. Staub, SPheno 3.1: extensions including flavour, CPphases and models beyond the MSSM. Comput. Phys. Commun. 183, 2458-2469 (2012). arXiv:1104.1573

106. W. Porod, SPheno, a program for calculating supersymmetric spectra, SUSY particle decays and SUSY particle production at e+ e-colliders. Comput. Phys. Commun. 153, 275-315 (2003). arXiv:hep-ph/0301101

107. L.U.X. Collaboration, D.S. Akerib et al., Results from a search for dark matter in the complete LUX exposure. Phys. Rev. Lett. 118(2), 021303 (2017). arXiv: 1608.07648

108. PandaX-II Collaboration, X. Cui et al., Dark matter results from 54-ton-day exposure of PandaX-II experiment. Phys. Rev. Lett. 119(18), 181302 (2017) arXiv:1708.06917

109. R. Jinno, M. Takimoto, Gravitational waves from bubble collisions: an analytic derivation. Phys. Rev. D 95(2), 024009 (2017). arXiv: 1605.01403

110. R. Jinno, M. Takimoto, Gravitational Waves from Bubble Dynamics: Beyond the Envelope. arXiv:1707.03111

111. K. Hashino, R. Jinno, M. Kakizaki, S. Kanemura, T. Takahashi, M. Takimoto, Fingerprinting Models of First-Order Phase Transitions by the Synergy Between Collider and Gravitational-Wave Experiments. arXiv:1809.04994 